\section{Comparison of time domain Stratus OCT and spectral domain SLO/OCT for assessment of macular thickness and volume}

R Forte, GL Cennamo, ML Finelli and

$G$ de Crecchio

\begin{abstract}
Purpose To compare retinal thickness and volume measurements obtained with Stratus time domain optical coherence tomography (OCT) and spectral domain scanning laser ophthalmoscope OCT (SD-SLO/OCT).

Methods In a cross-sectional study, 52 eyes with a normal macula, 30 eyes with retinal oedema, and 10 eyes with a myopia higher than $6 \mathrm{D}$ have been evaluated with both time domain OCT (TD-OCT, Stratus OCT, Carl Zeiss Meditec, USA) and SD-SLO/OCT (OTI, Toronto, Canada). Retinal thickness and volume measurements in the nine areas of the 6-mm ETDRS ring were compared. Artefacts were defined as the discordance between the automatically detected anterior and posterior retinal boundaries and the boundaries detected by the examiner.

Results Artefacts were more frequent with TD-OCT (35 vs 26\%). Mean retinal thickness was significantly higher with SD-SLO/OCT by $30.1 \mu \mathrm{m}( \pm 25.8)(P=0.003)$ in presence of the artefacts and by $39.2 \mu \mathrm{m}( \pm 25.8)(P=0.003)$ after their exclusion. The correlation between the two retinal thickness data sets before exclusion of the artefacts $(r=0.59, P<0.001)$ increased after their removal $(r=0.84$, $P<0.001)$. A strong correlation was present between the two retinal volume data sets before $(r=0.94, P<0.001)$ and after exclusion of the artefacts $(r=0.96, P<0.001)$.

Conclusions SD-SLO/OCT produced fewer artefacts than Stratus TD-OCT. This could be attributed to the greater resolution and acquisition speed of SD-SLO/OCT. The macular retinal thickness values measured with SD-SLO/OCT were significantly higher
\end{abstract}

than those measured with Stratus TD-OCT.

Retinal volumes measured with Stratus TD-OCT and SD-SLO/OCT were strongly correlated.

Eye (2009) 23, 2071-2078; doi:10.1038/eye.2008.363; published online 12 December 2008

Keywords: macular thickness; optical coherence tomography; spectral domain OCT; time domain OCT

\section{Introduction}

Traditional optical coherence tomography (OCT) is a time domain OCT (TD-OCT), ${ }^{1-4}$ where depth informations of the retina are obtained after a longitudinal translation in time of a reference arm. Good reproducibility of retinal thickness measurements by Stratus OCT (Carl Zeiss Meditec, USA) has been shown. ${ }^{5,6}$

Nevertheless, reproducibility may be increased by considering parameters that average more points (such as the total macular volume). ${ }^{7}$ Recently introduced spectral domain OCT (SDOCT) measures the interferometric signal detected as a function of optical frequencies, allowing for imaging speeds 50 times faster than TD-OCT, and providing a greater number of images per unit area. ${ }^{8-12}$ This increase in resolution and scanning speed allows highdensity raster scanning of retinal tissue while minimizing eye motion artefacts. A better repeatability of measurement compared with Stratus OCT has been reported for spectral domain OCT in healthy eyes. ${ }^{13,14}$ The presence of both time domain and spectral domain OCT systems in ophthalmic practices is becoming increasingly widespread, and the several
Eye Department, University Federico II, Naples,

Italy

Correspondence: R Forte, Eye Department, University Federico II, Via Pansini 5, Naples 80131, Italy Tel: +390817462293; Fax: +390817462383 E-mail: raifor@hotmail.com

Received: 20 May 2008 Accepted in revised form: 26 September 2008 Published online: 12 December 2008

Financial interest: None 
SD-OCT devices introduced to clinical practice use different algorithms to calculate retinal thickness. This is the first study where an evaluation of the artefacts and a comparison of retinal thickness and volume measurements obtained with both Stratus TD-OCT and OTI (Ophthalmic Technologies Inc., Canada) SD-OCT were performed in normal and pathologic eyes.

\section{Materials and methods}

In this cross-sectional observational study we considered 92 eyes (46 patients) evaluated from July to November 2007 in the Eye Department of the University Federico II of Naples. Fifty-two eyes of 26 volunteers without retinal abnormalities, 30 eyes (15 patients) with retinal oedema (18 with cystoid macular oedema, 8 with epiretinal membrane, 4 with both abnormalities), and 10 eyes (5 patients) with a myopia higher than 6D (range: 9-16D) were included in the study. This study has been performed in accordance with the ethical standards laid down in the 1964 Declaration of Helsinki. A written informed consent was obtained from all participants in this study. The study was approved by the ethics committee of the University Federico II, Naples, Italy.

\section{Optical coherence tomography}

Examination with time domain OCT and spectral domain OCT was performed on each eye on the same day and by the same examiners (RF or GC).

Time domain Stratus OCT (Stratus OCT, Version 4.0.1, Carl Zeiss Meditec, Dublin, CA, USA) uses a superluminescent diode source (wavelength $810 \mathrm{~nm}$, bandwidth $20 \mathrm{~nm}$ ) and generates retinal thickness map from six consecutive radial B-scans. In this study highresolution Radial Line scan protocol (1.0 B-scans/s, 512 axial measurements with a resolution of around $10 \mu \mathrm{m}$ ) was used to minimize artefacts. Central foveal thickness is calculated at the intersection of the six radial lines and is measured as the distance between an inner retinal boundary, represented by the internal limiting membrane (ILM), and an outer retinal boundary, represented by the junction between the inner and outer photoreceptor segments (IS/OS) ${ }^{15}$ Volume data (cubic millimeters) are obtained by multiplying the average thickness by the sampled area.

In spectral domain scanning laser ophthalmoscope/ OCT (SLO/OCT, Ophthalmic Technologies Inc., Toronto, Canada) a super luminescent diode produces a light with a wavelength of $840 \mathrm{~nm}$ (bandwidth $150 \mathrm{~nm}$ ). A spectrometer measures the interference spectrum between back reflected light from the tissue and light from a stationary reference arm. The Fourier transform of this interference spectrum allows to measure the magnitude and echo time delay of the light signal from the tissue. This provides simultaneous measurements of light echoes from different axial depths, without movement of the reference arm. Therefore, an improved acquisition speed is allowed. In SD-SLO/OCT the inner retinal boundary used to obtain retinal thickness is the ILM, whereas the exact location of the outer boundary is still unknown. When acquiring retinal topographic maps SD-SLO/OCT performs a sweep of 256 serial parallel OCT B-scans/s with an axial resolution of $5 \mu \mathrm{m}$, covering a $9 \times 9 \mathrm{~mm}$ area in the transverse plane through the macula, and providing the thickness of any point in the evaluated area. By this way, mean RT can be obtained, together with the RT measured in each point of the map. Retinal volume in a given area is obtained by the integral of the thickness measured in each point of the area.

In each eye considered in this study, mean retinal thickness (RT) and mean retinal volume (RV) were measured with both Stratus TD-OCT and SD-SLO/OCT in the nine macular Early Treatment Diabetic Retinopathy Study (ETDRS) areas. ETDRS areas include a central 1-mm disc, representing the foveal area, and inner and outer rings of 3 and $6 \mathrm{~mm}$, respectively. The inner and outer rings are divided into four quadrants: superior, nasal, inferior, and temporal. Central foveal thickness (FT) was also calculated. The total macular thickness (MT) and the total macular volume (MV) in the 6-mm ETDRS ring were calculated based on the proportional contribution of the regional macular thicknesses as described earlier. ${ }^{5,16}$

\section{Artefacts}

Evaluation of artefacts was performed for both Stratus TD-OCT B-scans and SD-SLO/OCT B-scans. We considered each of the six radial B-scans for Stratus TDOCT and the B-scans showed on the retinal map window for SD-SLO/OCT. Artefacts were defined as the discordance between the automatically detected anterior and posterior retinal boundaries and the boundaries detected by the examiner. ${ }^{15}$ Even slight discordances were considered as an artefact.

\section{Statistical analysis}

On the two sets of 1748 (RT, 920; RV, 828) values a quadrant-by-quadrant paired comparison was performed. The difference for FT, for mean RT and for mean RV in each ETDRS area, for MT and MV in the 6-mm ETDRS ring before and after exclusion of the artefacts was calculated, and results were expressed as means \pm standard deviation $( \pm S D$ ). Correlation between the means of the two data sets was obtained using paired samples $t$-test. Bland-Altman plots were used to assess 
their agreements. Regression analysis was estimated with Pearson's correlation coefficient $(r) . P<0.05$ was considered statistically significant. Statistical analysis was performed with SPSS software (version 13.0; SPSS Inc., Chicago, IL, USA) and MedCalc software (version 9.2; MedCalc Software, Mariakerke, Belgium).

\section{Results}

Before exclusion of the artefacts a statistically significant difference was present in each ETDRS quadrant between mean measurements obtained with the two OCT systems, except for temporal inner and nasal inner macula (Table 1). The correlation of MT measurements in each group before and after exclusion of artefacts is shown in Figure 1.

With regard to the data sets acquired in the 6- $\mathrm{mm}-$ diameter area in all eyes, the mean difference between the SD-SLO/OCT and TD-OCT macular thickness measurements in the presence of the artefacts was $30.1 \mu \mathrm{m}( \pm 59.5)(P<0.001)$ (Table 2$)$; the greater the thickness, the greater the difference $(20.5 \pm 25.3 \mu \mathrm{m}$ when $\mathrm{MT} \leqslant 250 ; 35.4 \pm 27.8 \mu \mathrm{m}$ when $\mathrm{MT}>250 \mu \mathrm{m})$. After exclusion of the artefacts the mean difference of MT was $39.2 \mu \mathrm{m}( \pm 25.8)(P=0.003)$, the greater the thickness, the greater the difference $(28.7 \pm 32.3 \mu \mathrm{m}$ when $\mathrm{MT} \leqslant 250 \mu \mathrm{m}$;
$47 \pm 37.2 \mu \mathrm{m}$ when $\mathrm{MT}>250 \mu \mathrm{m})$. The ratio between mean MT measured with SD-SLO/OCT and mean MT measured with Stratus TD-OCT in presence of artefacts was $1.12( \pm 0.4)$, and became $1.25( \pm 0.3)$ after exclusion of the artefacts (Figure 2). The correlation between the MT measurements with TD-OCT and SD-SLO/OCT in presence of artefacts $(r=0.59 ; P<0.001)$ increased after their exclusion $(r=0.84 ; P<0.001)$. With respect to $\mathrm{MV}$, a statistically significant difference was present before $(P=0.004)$ and after $(P<0.001)$ exclusion of the artefacts. A strong correlation was found between the two volume data sets before $(r=0.94 ; P<0.001)$ and after exclusion of the artefacts $(r=0.96 ; P<0.001)$.

In the group of eyes without retinal abnormalities the correlation between the two instruments in the measurement of MT was low before exclusion of the artefacts $(r=0.34 ; P<0.001)$ and increased after their removal $(r=0.88 ; P<0.001)$. Difference between mean MV did not show statistical significance before exclusion of the artefacts $(P=0.08)$, whereas it was significant after their removal $(P<0.001)$. A strong agreement was found in the measurement of MV before $(r=0.69 ; P<0.001)$ and after $(r=0.82 ; P<0.001)$ exclusion of the artefacts.

Among all the eyes presenting with retinal oedema, the correlation between the two instruments in measurement of MT was low before exclusion of the

Table 1 Summary of the mean retinal thickness ( \pm SD), retinal volume, and Pearson's correlation coefficient $(r)$ taken in all eyes in the nine macular Early Treatment Diabetic Retinopathy Study (ETDRS) areas with Stratus time domain OCT (TD-OCT) and OTI spectral domain SLO/OCT (SD-SLO/OCT)

\begin{tabular}{|c|c|c|c|c|}
\hline \multirow[t]{2}{*}{ ETDRS area } & \multicolumn{2}{|c|}{ Mean retinal thickness $(\mu m)$} & \multirow[t]{2}{*}{ P-values } & \multirow[t]{2}{*}{$\mathrm{r}$} \\
\hline & $T D-O C T$ & $S D-S L O / O C T$ & & \\
\hline Fovea & $256.6( \pm 99)$ & $281( \pm 166)$ & 0.07 & 0.62 \\
\hline Temporal inner macula & $290( \pm 80)$ & $300( \pm 131)$ & 0.4 & 0.6 \\
\hline Superior inner macula & $291( \pm 75)$ & $323( \pm 145)$ & 0.04 & 0.37 \\
\hline Nasal inner macula & $296.7( \pm 81)$ & $332.7( \pm 135)$ & 0.004 & 0.63 \\
\hline Inferior inner macula & $301.4( \pm 89)$ & $393.2( \pm 76)$ & 0.2 & 0.31 \\
\hline Temporal outer macula & $246.7( \pm 71)$ & $258.8( \pm 121)$ & 0.057 & 0.68 \\
\hline Superior outer macula & $253.6( \pm 58)$ & $316.7( \pm 132)$ & $<0.001$ & 0.62 \\
\hline Nasal outer macula & $274.9( \pm 73)$ & $302.7( \pm 124)$ & 0.057 & 0.5 \\
\hline \multirow[t]{3}{*}{ Inferior outer macula } & $263.8( \pm 94)$ & $295.5( \pm 134)$ & 0.009 & 0.69 \\
\hline & \multicolumn{2}{|c|}{ Retinal volume $\left(\mathrm{mm}^{3}\right)$} & & \\
\hline & TD-OCT & $S D-S L O / O C T$ & & \\
\hline Fovea & $0.2( \pm 0.07)$ & $0.2( \pm 0.12)$ & 0.5 & 0.6 \\
\hline Temporal inner macula & $0.4( \pm 0.12)$ & $0.4( \pm 0.2)$ & 0.7 & 0.6 \\
\hline Superior inner macula & $0.4( \pm 0.11)$ & $0.5( \pm 0.19)$ & 0.2 & 0.4 \\
\hline Nasal inner macula & $0.4( \pm 0.12)$ & $0.5( \pm 0.22)$ & 0.01 & 0.62 \\
\hline Inferior inner macula & $0.5( \pm 0.13)$ & $0.5( \pm 0.22)$ & 0.7 & 0.63 \\
\hline Temporal outer macula & $1.3( \pm 0.38)$ & $1.3( \pm 0.63)$ & 0.3 & 0.68 \\
\hline Superior outer macula & $1.3( \pm 0.3)$ & $1.6( \pm 0.6)$ & $<0.001$ & 0.73 \\
\hline Nasal outer macula & $1,4( \pm 0.4)$ & $1.5( \pm 0.7)$ & 0.1 & 0.51 \\
\hline Inferior outer macula & $1,5( \pm 0.9)$ & $1,5( \pm 0.7)$ & 0.8 & 0.38 \\
\hline Total volume & $7.39( \pm 2)$ & $8( \pm 3)$ & 0.01 & 0.71 \\
\hline
\end{tabular}



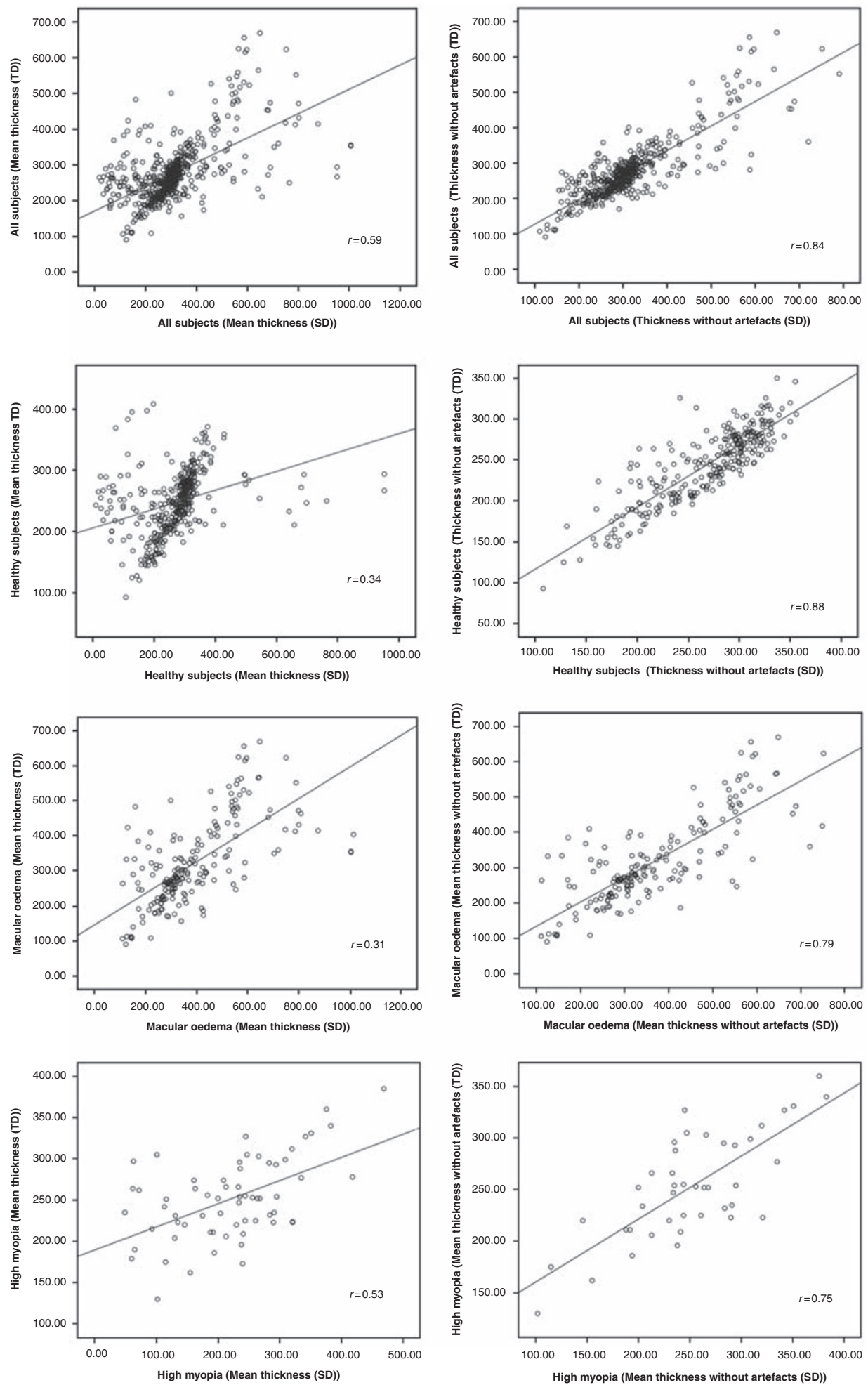

Figure 1 Graph showing the correlation between mean macular thickness measurements $(\mu \mathrm{m})$ acquired in the 6-mm-diameter area with time domain OCT ( $Y$-axis) and spectral domain SLO/OCT (X-axis) in the four groups before and after exclusion of artefactual quadrants (TD, time domain; SD, spectral domain; $r=$ Pearson's correlation coefficient). 
Table 2 Mean macular thickness ( \pm SD) in the 6-mm-diameter area, as measured with Stratus time domain OCT (TD-OCT) and OTI spectral domain SLO/OCT (SD-SLO/OCT) in all patients and in the three subgroups before and after exclusion of artefacts

\begin{tabular}{|c|c|c|c|c|c|}
\hline & Stratus OCT & $S D S L O / O C T$ & $\begin{array}{c}\text { Mean difference } \\
\text { (SD SLO/OCT-TD OCT) }\end{array}$ & P-values & $\mathrm{r}$ \\
\hline All eyes with artefacts & $275.5( \pm 81)$ & $305.6( \pm 140.5)$ & 30.1 & $<0.001$ & $0.59(P<0.001)$ \\
\hline All eyes without artefacts & $274.6( \pm 81.1)$ & $313.8( \pm 105.6)$ & 39.2 & $<0.001$ & $0.84(P<0.001)$ \\
\hline Normal eyes with artefacts & $247( \pm 51)$ & $281( \pm 88)$ & 34 & $<0.001$ & $0.34(P<0.001)$ \\
\hline Normal eyes without artefacts & $246.2( \pm 50)$ & $277.1( \pm 66)$ & 30.9 & $<0.001$ & $0.88(P<0.001)$ \\
\hline Macular oedema with artefacts & $329.4( \pm 120.7)$ & $401.8( \pm 449.1)$ & 72.4 & 0.002 & $0.31(P<0.001)$ \\
\hline Macular oedema without artefacts & $319.6( \pm 119.7)$ & $383.6( \pm 170.3)$ & 64 & $<0.001$ & $0.79(P<0.001)$ \\
\hline High myopia with artefacts & $248.8( \pm 48.9)$ & $216.8( \pm 94.3)$ & -32 & 0.003 & $0.53(P<0.001)$ \\
\hline High myopia without artefacts & $253( \pm 50.8)$ & $251.7( \pm 63)$ & -1.3 & 0.1 & $0.75(P<0.001)$ \\
\hline
\end{tabular}

$r=$ Pearson's correlation coefficient.
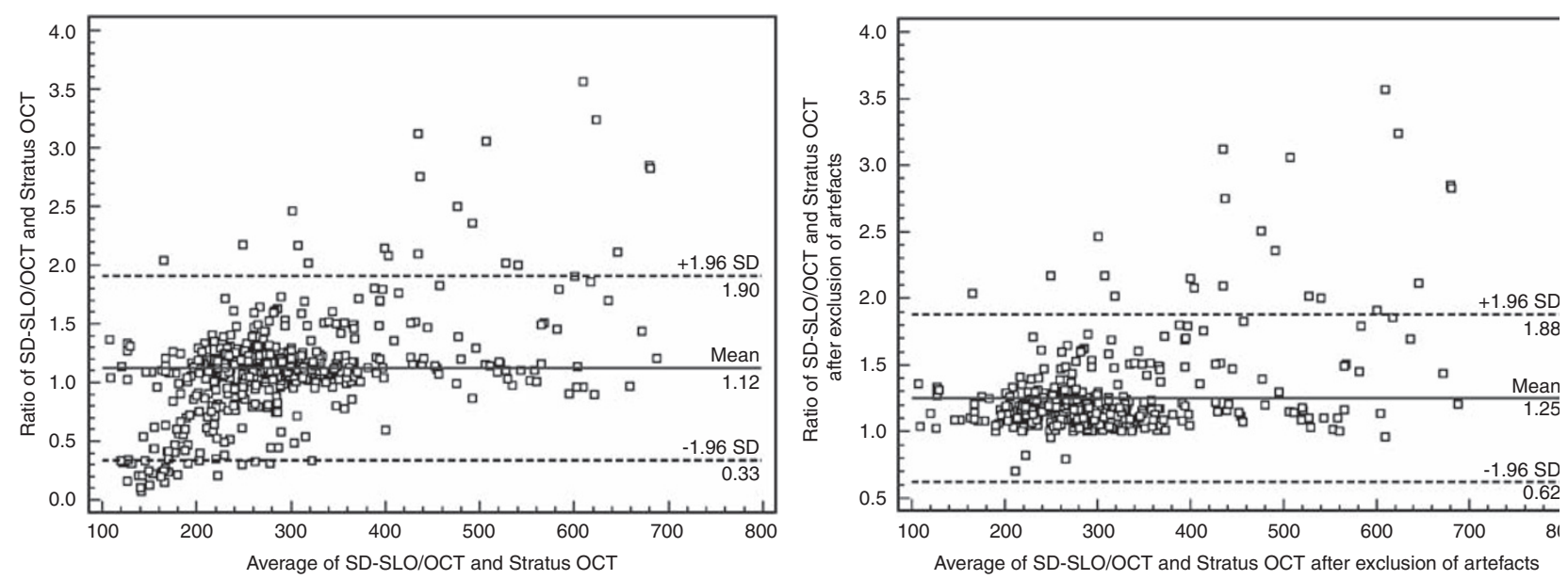

Figure 2 Bland-Altman plot showing the ratio between mean macular thickness measurements with Stratus OCT and spectral domain SLO/OCT (SD-SLO/OCT) against the average of the two $(\mu \mathrm{m})$ in all eyes, before (left) and after (right) exclusion of the artefacts.

artefacts $(r=0.31 ; P<0.001)$ and increased after their removal $(r=0.79 ; P<0.001)$. As concerns evaluation of MV a statistically significant difference was found before $(P<0.001)$ and after $(P=0.001)$ exclusion of the artefacts. A strong agreement was found before $(r=0.95 ; P<0.001)$ and after $(r=0.96 ; P<0.001)$ exclusion of the artefactual quadrants.

In the 10 eyes presenting with high myopia, MT was higher with TD-OCT than with SD-OCT before and after removal of the artefacts. Before exclusion of the artefacts the MT difference was significant $(P=0.003)$. A low correlation was found between the two groups in evaluation of MT before exclusion of the artefacts $(r=0.53 ; P<0.001)$ whereas a higher agreement was found after their removal $(r=0.75 ; P<0.001)$. In the same group, a statistically significant difference between mean MV was present among the two data sets before exclusion of the artefacts $(P=0.01)$, whereas after their exclusion the difference between mean MV was not statistically significant, although a trend towards significance was noted $(P=0.07)$. There was a strong correlation in evaluation of MV before $(r=0.92 ; P<0.001)$ and after $(r=0.92 ; P<0.001)$ exclusion of the artefactual quadrants.

\section{Artefacts}

Of the two pairs of 92 acquisition values, 40/184 (21.7\%) exhibited artefacts with either Stratus TD-OCT or SDSLO/OCT or both. With Stratus TD-OCT, artefacts were present in 32/92 cases (35\%) (in 4 cases with healthy retina, in 18 with retinal oedema, and in the 10 eyes with high myopia). Among highly myopic eyes they involved the $3-\mathrm{mm}$ central area in 8/10 cases. With SD-SLO/OCT, artefacts were present in 24/92 cases (26\%) (in 2 cases with healthy retina, in 12 with retinal oedema, and in the 
10 eyes with high myopia). Among highly myopic eyes the 3-mm central area was never affected by artefacts after evaluation with SD-SLO/OCT.

Artefacts that determined an overestimation of RT and $\mathrm{RV}$ were due to an erroneous positioning of the inner retinal boundary, after the presence of an epiretinal membrane (8/40 cases, 20\%) and detached posterior hyaloid (11/40 cases, $27.5 \%)$. Artefacts that determined an underestimation of RT and RV were due to the presence of intraretinal hard exudates in 15/40 cases (37.5\%), and due to intraretinal cysts in 6/40 cases (15\%) with cystoid oedema, whose boundaries were interpreted as outer retinal layers.

\section{Discussion}

This cross-sectional case study is, to our knowledge, the first reported quantitative comparison of retinal thickness and volume measurements obtained with a TD-OCT system and SD-OCT system in normal, oedematous, and highly myopic eyes. By comparing macular thickness and volume, we assessed the intersystem reproducibility of two widely used indicators of the retinal integrity.

The number of artefacts obtained with SD-OCT ( $26 \%$ of the acquisitions) was lower than that obtained with TD-OCT (35\% of the acquisitions). The correlation between the macular thickness data sets significantly increased after the exclusion of the artefacts. An inaccurate recognition of the retinal inner and outer boundaries determines the presence of the artefacts and influences retinal thickness measurements. Boundary recognition is related to the resolution of the system. The axial resolution of OCT is inversely related to the spectral bandwidth of the optical source, via the formula: resolution $=2 \ln (2) \lambda 2 /(\pi \Delta \lambda)$, where $(\Delta \lambda)$ is the bandwidth and $(\lambda)$ is the central wavelength of the light source. ${ }^{10}$ Therefore, the broader the wavelength distribution within the incident beam, the higher the resolution. Time-domain, standard-resolution, commercially available OCT systems use superluminescent diodes emitting light with $20-30 \mathrm{~nm}$ bandwidths centered at $830 \mathrm{~nm}$, resulting in a $10-15 \mu \mathrm{m}$ axial image resolution. Spectral SLO/OCT system employs a compact superluminescent diode with a $150 \mathrm{~nm}$ bandwidth centered at $890 \mathrm{~nm}$, which results in a $5 \mu \mathrm{m}$ axial resolution. The presence of artefacts is also influenced by the acquisition speed. The speed of the commercially available Stratus TD-OCT system $(0.5-1.0 \mathrm{~B}$-scan/s) is considerably lower than that of SD-SLO/OCT (256 serial parallel OCT B-scans/s). Furthermore, in SD-SLO/OCT system the high acquisition speed increases signal to noise ratio.
Therefore, the reduced number of artefacts obtained with SD-OCT could be attributed to its greater resolution, acquisition speed, and signal to noise ratio.

Except for highly myopic eyes, the retinal thickness values measured with SD-SLO/OCT were significantly higher than those measured with Stratus TD-OCT, the greater the thickness, the greater the difference. The ratio between the mean retinal thickness measurement with SD-SLO/OCT and the mean retinal thickness measurement with Stratus TD-OCT in all considered eyes was 1.1 before exclusion of the artefacts and 1.2 after their removal. The poor agreement between Stratus OCT and a different spectral domain OCT (3D OCT, Topcon, Tokyo, Japan) has already been reported. ${ }^{13}$ Although this was limited to normal eyes, it resulted in a SD-OCT/ TD-OCT ratio of 1.01 and a correlation between the amount of difference and the thickness was not suggested. To obtain retinal thickness measurement, Stratus OCT uses segmentation algorithms to mark the ILM as the inner boundary and the inner segment/outer segment junction (IS/OS) as the outer boundary. ${ }^{15}$ To date, no data are provided about boundary recognition in the OTI SD-SLO/OCT system. The higher RT measured with OTI SD-SLO/OCT in this study is likely due to detection of a more external outer boundary. The different resolution and the different algorithms of SDOCTs, the reduction of motion artefacts, and the absence of interpolation could determine the detection of a different outer boundary, such as the retinal pigment epithelium or the intermediate reflective line, which is thought to represent the tip of the cone outer segment in the fovea. ${ }^{17}$ Furthermore, as the segmentation algorithms are different among the SD-OCT devices, the outer boundary used for thickness measurement may strongly depend on the OCT system used. Thus, retinal thickness measurements will consequently differ between both TDOCT and SD-OCT and among different SD-OCT systems.

In all areas a larger standard deviation was present after thickness measurement with SD-OCT. This could be explained by the different ways with which the OCT systems measure retinal thickness. Stratus OCT uses six radial B-scans and mathematical interpolations are used to derive thickness estimations for the spaces in between. On the other hand, SD-SLO/OCT evaluates every single point in the sampled area, with a consequently larger standard deviation.

After evaluation with SD-OCT in the group with macular oedema, the standard deviation of MT in the 6 - $\mathrm{mm}$ area before exclusion of the artefacts $( \pm 449.1)$ was considerably larger than after exclusion $( \pm 170.3)$, whereas the difference between mean macular thickness values was less considerable. In macular oedema the presence of intraretinal exudates, intraretinal cysts or epiretinal membrane may affect the right recognition of 
inner and outer boundaries by the system, with a consequent increase of the artefacts and with erroneous thickness measurements. As SD-OCT measures retinal thickness in every single point, the presence of the artefacts will greatly influence the standard deviation, whereas the effect on the mean thickness in the considered area will be less evident.

In highly myopic eyes, artefacts were present in all cases at examinations with both Stratus TD-OCT and SD-SLO/OCT, and significantly influenced the correlation between the thickness measurements. Artefacts in high myopia are likely because of axial elongation and retinal pigment layer thickening, with consequent misdetection of the retinal boundaries by the OCT system. In the SD-SLO/OCT data set artefacts were always distributed out of the central 3-mm area, forasmuch as the myopic bulb elongation could likely limit the capacity of the focusing system to follow the retinal boundaries out of the fovea.

A significant difference in retinal thickness measurements was present in myopic eyes between Stratus TD-OCT and SD-SLO/OCT before exclusion of the artefacts, whereas after exclusion the difference was not significant. Furthermore, in highly myopic eyes the MT evaluated with TD-OCT was higher than MT measured with SD-SLO/OCT. This is in contrast with the significantly higher retinal thickness found with SD-SLO/OCT in emmetropic eyes before and after exclusion of the artefacts. Ocular stretching of highly myopic eyes determines the hyperreflective outer lines to be very close to each other. The retinal thickness maps rely on a computer algorithm to segment the inner and outer boundaries of the retina, and these algorithms may fail to identify the boundaries if the retinal architecture is distorted. Therefore, as in high myopia the IS/OS is often not visible, both TD- and SD-OCT systems may find a similar outer boundary, with a consequently reduced difference between the two systems in thickness measurement.

In this study, we found a strong correlation between volume measurements obtained with TD- and SD-OCT. This correlation was not influenced by the presence of the artefacts. Total retinal volume has been suggested as a parameter to be considered to increase intervisit reproducibility in Stratus OCT, as it samples greater areas of the macula. ${ }^{7}$ To obtain retinal volume in a given area, Stratus TD-OCT multiplies the mean retinal thickness by that area, whereas SD-SLO/OCT calculates the integral of the thicknesses in the same known area. Although the thickness measurements in each point may differ between the two OCT systems, the evaluated area is constant. This could explain the strong correlation found in our series in all the evaluated quadrants. As retinal thickness comparison seems more influenced by the segmentation algorithm used by the OCT device, the retinal volume could represent a point of reference when comparing retinal maps obtained with Stratus TD-OCT and SD-SLO/OCT.

This study has the limitation of evaluating only two OCT devices, each one representing a different OCT imaging technology. Several SD-OCT systems with different proprietary segmentation algorithms are being introduced to ophthalmologists and Stratus OCT is not the only commercially available TD-OCT. The ratio between MT measured with SD-SLO/OCT and TD-OCT was 1.1, whereas it has resulted to be 1.01 in case of a different SD-OCT device. ${ }^{13}$ However, our data show that the different OCT acquisition systems determine different retinal thickness measurements but scarcely influence retinal volume measurements.

In conclusion, SD-SLO/OCT produced fewer artefacts than Stratus TD-OCT in eyes with normal and oedematous retina. In highly myopic eyes the overall number of artefacts was the same, although with SDSLO/OCT they never involved the central 3-mm area. Retinal thickness obtained with SD-SLO/OCT was significantly greater than retinal thickness measured with Stratus TD-OCT. Therefore, when following patient progress the use of a single OCT device seems advisable, otherwise a correcting value of 1.1 should be considered when extrapolating retinal thickness measurements from Stratus TD-OCT to SD-SLO/OCT. Retinal volume measurements showed a strong reproducibility and could be used to compare examinations with Stratus TD-OCT and SD-SLO/OCT. Further studies comparing different TD- and SD-OCT devices are needed to assess the general validity of our findings.

\section{References}

1 Huang D, Swanson EA, Lin CP, Schuman JS, Stinson WG, Chang W et al. Optical coherence tomography. Science 1991; 254(5035): 1178-1181.

2 Hee MR, Izatt JA, Swanson EA, Huang D, Schuman JS, Lin $\mathrm{CP}$ et al. Optical coherence tomography of the human retina. Arch Ophthalmol 1995; 113: 325-332.

3 Puliafito CA, Hee MR, Lin CP, Reichel E, Schuman JS, Duker JS et al. Imaging of macular diseases with optical coherence tomography. Ophthalmology 1995; 102(2): 217-229.

4 Thomas D, Duguid G. Optical coherence tomography-a review of the principles and contemporary uses in retinal investigation. Eye 2004; 18(6): 561-570.

5 Paunescu LA, Schuman JS, Price LL, Stark PC, Beaton S, Ishikawa $\mathrm{H}$ et al. Reproducibility of nerve fiber thickness, macular thickness, and optic nerve head measurements using StratusOCT. Invest Ophthalmol Vis Sci 2004; 45(6): 1716-1724.

6 Gurses-Ozden R, Teng C, Vessani R, Zafar S, Liebmann JM, Ritch R. Macular and retinal nerve fiber layer thickness measurement reproducibility using optical coherence tomography (OCT-3). J Glaucoma 2004; 13(3): 238-244. 
7 Joeres S, Tsong JW, Updike PG, Collins AT, Dustin L, Walsh $\mathrm{AC}$ et al. Reproducibility of quantitative optical coherence tomography subanalysis in neovascular age-related macular degeneration. Invest Ophthalmol Vis Sci 2007; 48(9): 4300-4307.

8 Drexler W, Sattmann H, Hermann B, Ko TH, Stur M, Unterhuber A et al. Enhanced visualization of macular pathology with the use of ultrahigh-resolution optical coherence tomography. Arch Ophthalmol 2003; 121(5): 695-706.

9 Wojtkowski M, Srinivasan V, Fujimoto JG, Ko T, Schuman JS, Kowalczyk A et al. Three-dimensional retinal imaging with high-speed ultrahigh-resolution optical coherence tomography. Ophthalmology 2005; 112(10): 1734-1746.

10 Wojtkowski M, Srinivasan VJ, Ko TH, Fujimoto, Kowalczyk A, Duker J. Ultrahigh-resolution, high-speed, Fourier domain optical coherence tomography and methods for dispersion compensation. Opt Express 2004; 12: 2404-2422.

11 Ko TH, Fujimoto JG, Duker JS, Paunescu LA, Drexler W, Baumal CR et al. Comparison of ultrahigh- and standardresolution optical coherence tomography for imaging macular hole pathology and repair. Ophthalmology 2004; 111(11): 2033-2043.

12 Koizumi H, Spaide RF, Fisher YL, Freund KB, Klancnik Jr JM, Yannuzzi LA. Three-dimensional evaluation of vitreomacular traction and epiretinal membrane using spectral-domain optical coherence tomography. Am J Ophthalmol 2008; 145(3): 509-517.

13 Leung CK, Cheung CY, Weinreb RN, Lee G, Lin DS, Pang $\mathrm{CP}$ et al. Comparison of macular thickness measurements between time domain and spectral domain optical coherence tomography. Invest Ophthalmol Vis Sci 2008; 49(11): 4893-4897.

14 Forooghian F, Cukras C, Meyerle CB, Chew EY, Wong WT. Evaluation of time domain and spectral domain optical coherence tomography in the measurement of diabetic macular edema. Invest Ophthalmol Vis Sci 2008; 49(10): 4290-4296.

15 Pierre-Kahn V, Tadayoni R, Haouchine B, Massin P, Gaudric A. Comparison of optical coherence tomography models OCT1 and Stratus OCT for macular retinal thickness measurement. Br J Ophthalmol 2005; 89(12): 1581-1585.

16 Leung CK, Chan WM, Yung WH, Ng AC, Woo J, Tsang MK et al. Comparison of macular and peripapillary measurements for the detection of glaucoma: an optical coherence tomography study. Ophthalmology 2005; 112(3): 391-400.

17 Matsumoto H, Kishi S, Otani T, Sato T. Elongation of photoreceptor outer segment in central serous chorioretinopathy. Am J Ophthalmol 2008; 145(1): 162-168. 